\title{
Dosimetric Comparison Of Forward and Inverse Intensity Modulated Radiation Therapy Planning, And Volumetric Modulated Arc Therapy for (6 and10) MV x-ray Photons For Left Breast Cancer
}

\author{
Zeinab E. Hassan ${ }^{(1)}$,Mahmoud M. Ahmed ${ }^{(1)}$,Anwar A. Elsayed ${ }^{(2)}$ and Heba M. \\ Fahmy ${ }^{(2)}$ \\ (1) National Cancer Institute, Cairo University , Cairo, Egypt \\ (2) Biophysics Department, Faculty of Science, Cairo University ,Egypt.
}

\begin{abstract}
To study the best planning techniques for post operative breast radiotherapy either F-IMRT or I-IMRT and VMAT . Another strategy is to check the dosimetric difference between using 6 or $10 \mathrm{MV}$ energies for both I-IMRT and VMAT .

: In the present study, four different inverse plans and one forward plan of randomly selected twenty left breast cancer patients were compared dosimetrically.Plans were done on Monaco (5.1) treatment planning system and data analyses were accomplished using one-way Anova test using IBM SPSS (20) data editor software.

Inverse planning achieve superior target coverage over forward planning ( $\mathrm{p}$ value $=0.001$ , 0.07 ) and conformity index ( $p$ value $<0.05$ ) maintaining adequate homogeneity index ( $p$ value $=0.461,0.138)$. Left lung and heart high dose levels decreased using I-IMRT, VMAT ( $p$ value $<0.05$ ) at the cost of increasing volume irradiated by low doses ( $\mathrm{p}$ value $<0.05$ ). For contralateral lung VMAT increased absorbed dose over F-IMRT $(\mathrm{p}<0.05)$ but I-IMRT showed non significant increase of V5 GY ( $\mathrm{p}$ value $=0.14)$. For contralateral breast both I-IMRT and VMAT increased absorbed dose over F-IMRT $(\mathrm{P}<0.05)$.

It may be concluded that with inverse planning achieved better target coverage that increases tumor control . Inverse planning also achieved lower volume of high doses that reduces acute radiation effect and increased irradiated volume by low doses significantly that increases the probability of late radiation effect.
\end{abstract}

Key words: F-IMRT, I-IMRT, VMAT, treatment planning system TPS

\section{Introduction}

Breast cancer is the most occurring cancer among women. About 2.1 million cases are newly diagnosed as breast cancer cases each year and 627,000deaths among women each year worldwide( Arbyn et al ; 2020). Lung cancer is in the primary cause of cancer death, with an estimated 1.8 million deaths (18\%), followed by colorectal $(9.4 \%)$, liver $(8.3 \%)$, stomach $(7.7 \%)$, and female breast $(6.9 \%)$ cancers in the fourth stage ( Sung et al ; 2021).. Some clinical trials showed that combining breast -conserving surgery (BCS) with postoperative radiation therapy (PORT) ( Sardaro et al ; 2012 )gives similar cure rates as Halsted radical mastectomy(McLaughlin et al ; 2008 ). On the other hand, PORT increases the risks of heart damage (Hooning et al ; 2007), lung

Correspondsing author email: mahmoud.meselhy@nci.cu.edu.eg

Recevied 28/12/2020, accepted 6/4/2021

DOI : $10.21608 /$ ejbbe.2021.55538.1039

(C)2021 Nathional Information and Documentation Center (NIDOC) 
cancer(Hensonet al ; 2013 ) and increases risk of introducing new cancer in healthy tissues due to ionizing radiation (Morgan et al ; 2012 ).

Alot of recent developments result in revolution in radiation therapy techniques(Simõeset al ; 2018 ), dose calculation and optimization algorithms (Woon et al ; 2016 ) and treatment delivery depending on the usage of multi-leaf collimator (MLC)(Avigo et al ; 2017 ).Parallel to this revolution, , advances in breathing control can reduce cardiac doses by controlling breath phase during simulation and treatment, then gating radiation dose according to the best phase away of heart (de Almeida et al ; 2012 ) .All these factors lead to the introduction of different planning techniques like field in field conformal radiotherapy (Ercan et al ; 2010 )or forward intensity modulated radiotherapy (F- IMRT ) (Liu et al ; 2015 ), inverse intensity modulated radiotherapy (I-IMRT) ( Narayanasamy et al ; 2015 ), and volumetric modulated arc therapy ( VMAT ) ( Rangaraj et al ; 2010 ) . (Henry et al ; 2018 ) and (lauche et al ; 2016 )studied the dosimetric benefit of using helical tomotherapy (HT) in the treatment of breast cancer specially with complex and concave breast shapes. The dosimetric differences among these techniques related to breast cancer treatment are investigated for different beam energies.

The aim of the present study :

First is to compare the dosimetric differences among different plans; F- IMRT, I-IMRT and VMAT.

Second to study impact of using 6versus 10 $\mathrm{MV}$ in inverse planning on dose homogeneity, MU and scattered dose in patient.

\section{Materials and Methods}

\section{Patients'selection}

Randomly selected twenty left-sided breastconserving surgery (BCS) patients previously treated by F-IMRT are the target of this study.

\section{Computed tomography (CT) simulation}

$\mathrm{GE}^{\circledR} \quad$ (general electric) Light speed $^{\circledR} \mathrm{CT}$ scanner was used to obtain the CT scans. All the patients underwent conventional $\mathrm{CT}$ on flat table board. Patients were positioned supine with both arms above the head on Klarity ${ }^{\circledR}$ breast board, making chest horizontal as much as possible. Images were acquired from the top of the head to the mid-abdomen, using a 2.5 -mm slice thickness.

\section{Planning target volume (PTV) contouring}

The image sets were transferred to the ELEKTA Focal ${ }^{\circledR}$ system for contouring. The clinical target volume (CTV) breast included all the visible breast parenchyma. On each slice, the breast volume extended from the pectoralis major muscle to the skin, excluding the pectoralis muscle, the ribs and the first $5 \mathrm{~mm}$ of skin. The PTV-breast was expanded by $5 \mathrm{~mm}$ in all directions around the CTV-breast, cropped from the skin surface; including the set-up margin and patient movement (liu et al ; 2015).CTV and organs at risk (OAR) were contoured by same radiation oncologist to minimize variation between different observers in this planning study.

\section{Delineation of OARs}

The heart was contoured from the level of the pulmonary trunk to the apex and included the pericardium but not the major vessels. Both lungs were contoured by auto segmentation tool, and the contralateral breast manually delineated. The breast volume of twenty left breast cancer patients used ranged from $560-2043\left(\mathrm{~cm}^{3}\right)$.

\section{Treatment planning system and linear accelerator}

After patient delineation was completed, it was sent toELEKTA Monaco5.1 ${ }^{\circledR}$ treatment planning system (TPS) for planning. Plans were calculated using commissioning data of ELEKTA SYNERGY ${ }^{\circledR}$ linear accelerator with 80 -multileaf collimator with a projected width of $10 \mathrm{~mm}$ at the isocenter. ELEKTA SYNERGY delivers forward and inverse IMRT with 6 and $10 \mathrm{MV}$ photon energies. For inverse plans the minimum segment width was set in MONACO TPS at 5 $\mathrm{mm}$ with the minimum Monitor Units (MUs) of control points (CPs) at $4 \mathrm{MU}$, the final dose calculation and segment optimization used the X-ray Voxel Monte Carlo (XVMC) algorithm with a calculation grid of $3 \mathrm{~mm}$ and $1 \%$ standard deviation (Jabbari et al ; 2011 ). MONACO uses fast and accurate collapsed cone dose calculation algorithm for forward plans.

A total dose of 40.05 Gy over 15 fractions for the whole breast was prescribed. The dose constraints of the planning target volume (PTV) and organs at risk (OAR) followed radiation therapy oncology group (Chen et al ; 2015 ) are listed in Table (1):

\section{Planning process}

Forward IMRT planning

Two open medial and lateral tangential beams open around PTV by a margin of $0.5 \mathrm{~cm}$ and open

Egypt. J. Biophys. Biomed. Eng., Vol. 22,No.1 (2021) 
TABLE 1. Dose constraints of the planning target volume (PTV) and organs at risk (OAR)followed radiation therapy oncology group (RTOG 1005)

\begin{tabular}{|c|c|c|}
\hline Parameter & Objective and constraint & Definition \\
\hline PTV V $95 \%$ & $95 \%$ of prescribed dose & Volume of PTV that receives $95 \%$ of prescribed dose \\
\hline PTV D 2\% & 46-48 Gy & Dose received by $2 \%$ of PTV volume \\
\hline PTV homogeneity index (HI) & Near zero as possible & (D2\%-D98\%) / D50 \% \\
\hline Monitor units (MU) & As low as possible & Output of machine \\
\hline Left lung V20 Gy & $16-20 \%$ & Volume of left lung that receives $20 \mathrm{~Gy}$ \\
\hline Right lung V 5 Gy & $10-15 \%$ & Volume of right lung that receives $5 \mathrm{~Gy}$ \\
\hline Heart V 10 Gy & $10-15 \%$ & Volume of heart that receives $10 \mathrm{~Gy}$ \\
\hline Heart D 5\% & $20-25$ Gy & Dose received by $5 \%$ of heart volume \\
\hline Heart mean dose & 4-5 Gy & Average dose received by heart \\
\hline contralateral breast (D max) & 3.1-4.96 Gy & Maximum point dose of right breast \\
\hline Contralateral breast (D 5\%) & $1.86-3.1 \mathrm{GY}$ & Dose received by $5 \%$ of right breast volume \\
\hline
\end{tabular}

$2 \mathrm{~cm}$ in air to take breathing process into account. Gantry angles for medial tangential beam was $310 \pm 10$ degree and lateral beam $130 \pm 10$, making lower border of beams parallel. Angles set to decrease volume irradiated of left lung and heart as possible and to avoid entrance and exit on contralateral breast. For each beam up to 2 fields in field were used to improve dose homogeneity as much as possible .Energy selection depends on case to make dose homogenous as much as possible either by $6 \mathrm{MV}$ or $10 \mathrm{MV}$ or mixing between 6 and $10 \mathrm{MV}$.

\section{Inverse IMRT planning}

Five different gantry angled beams were used and rotated around PTV following the medial and lateral borders of breast and set at angles (150100-40-0-310) and may differ slightly \pm 5 degrees according to case anatomy.Plan optimized one time by $6 \mathrm{MV}$ then energy changed to $10 \mathrm{MV}$ and re optimized .

\section{VMAT planning}

One partial arc with two rotations was used around PTV, following medial and lateral borders of breast. Gantry started at angle $150^{\circ}$, then rotated counterclockwise 220-degree and may differ slightly \pm 5 degrees according to case anatomy.
PTV objectives and OAR constraints were set on Monaco TPS to achieve the required criteria as stated in its manual. Plan optimized one time by $6 \mathrm{MV}$ then energy changed to $10 \mathrm{MV}$ and re optimized.

\section{Results}

All data analysis was performed according to one-way ANOVAs test in IBM SPSS (20) data editor. Each two planswere compared with each other and the statistical significance wascalculated. Results are considered significant if $\mathrm{P}$ value less than 0.05 . Dose distribution comparison at sagittal, coronal and transverse planes is represented in figure (1).

Homogeneity index (HI) and conformity index CI are calculated according to ICRU (50) formula HI $=$, and conformity index $\mathrm{CI}=$.

\section{PTV parameters}

Mean value of all PTV parameters (V95\%D2\%-CI-HI-MU) are shown in table (2). The volume that received more than $95 \%$ of the prescribed dose (V95\%) showed significant improve using 6MV I-IMRT (96.8 \pm 0.6825$)$ compared to F-IMRT $(92.96 \pm 0.894)(\mathrm{p}=0.001)$ and nearly significant with VMAT $(94.977 \pm 0.64)$ 


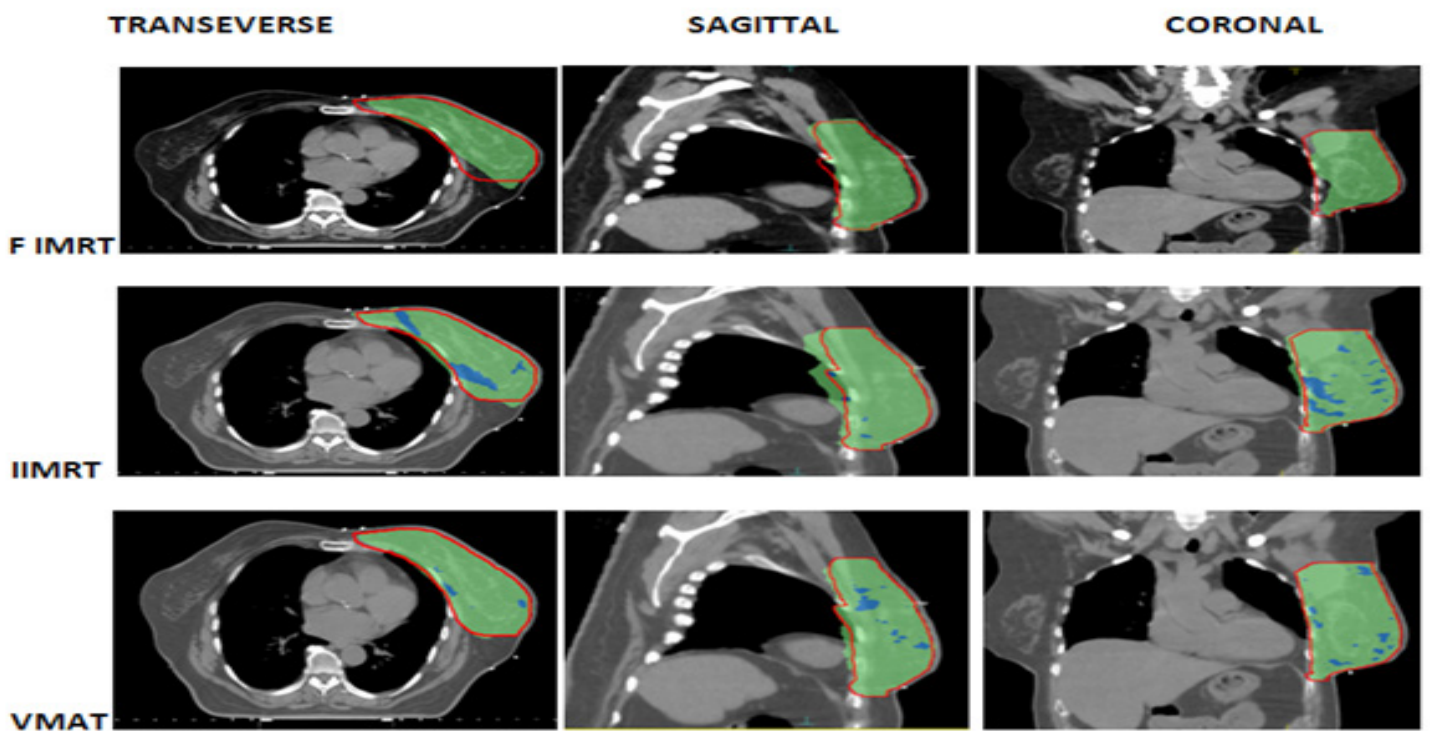

Fig. 1. Dose distribution of F-IMRT, I-IMRT, and VMAT respectively at three different planes green (95\% isodose line), blue (110\% isodose line), red PTV contour.

TABLE 2. value of all PTV parameters (V95\%-D2\%-CI-HI-MU) .( a ,d inverse and forward respectivelyb) intensity modulated radiation therapy ,c volumetric modulated arc therapy $, 1,2,3$ conformity indexhomogeneity index -and monitor units )

\begin{tabular}{cccccc}
\hline & $\begin{array}{c}\text { 6MV I }^{\mathbf{a}} \text { - } \\
\text { IMRT }^{\mathbf{b}}\end{array}$ & 10MV I-IMRT & 6MV VMAT $^{\mathbf{c}}$ & 10MV VMAT & F $^{\mathbf{d}}-\mathbf{I M R T}$ \\
\hline $\mathrm{V} 95 \%$ & $96.8 \pm 0.6825$ & $93.368 \pm 0.89$ & $94.977 \pm 0.64$ & $95.2 \pm 0.74$ & $92.96 \pm 0.894$ \\
$\mathrm{D} 2 \%$ & $45.1 \pm 0.1657$ & $44.4 \pm 0.17589$ & $44.482 \pm 0.154$ & $43.75 \pm 0.1354$ & $43.12 \pm 0.661$ \\
$\mathrm{CI}{ }^{1}$ & $0.85 \pm 0.01$ & $0.84 \pm 0.008$ & $0.89 \pm 0.01$ & $0.897 \pm 0.009$ & $0.71 \pm 0.1$ \\
$\mathrm{HI}^{2}$ & $0.18 \pm 0.013$ & $0.195 \pm 0.013$ & $0.195 \pm 0.013$ & $0.1627 \pm 0.01$ & $0.1698 \pm 0.007$ \\
$\mathrm{MU}^{3}$ & $726 \pm 16$ & $1070 \pm 45$ & $1111.7 \pm 30$ & $1176 \pm 25$ & $326 \pm 9$ \\
\hline
\end{tabular}

( $\mathrm{p}=0.07)$ compared to F-IMRT.Using higher energy for I-IMRT decreased V95\% significantly $(\mathrm{p}=0.002)$ and non-significantly for VMAT. So inverse planning improved PTV coverage but only with $6 \mathrm{MV}$. There was non-significant difference $(1.9 \%)$ between 6MV (I- IMRT) and $6 \mathrm{MV}$ (VMAT) $(\mathrm{p}=0.94)$ for target coverage. All I- IMRT plans $(p=0.007)$ and VMAT plans $(p=0.004)$ increased the dose received by 2 $\%$ of PTV volume (D2\%) more than F-IMRT planby about (4.6\% and $3.6 \%$ respectively) as shown in table (2). Different energies have nonsignificant effects on D2 \%( average 1.5\%) either with I- IMRT $(p=0.112)$ or VMAT $(p=0.121)$. So inverse planning increased hot spots by increasing D2\% and using higher energy don't decrease it significantly. Both I- IMRT and VMAT significantly improved the conformity index (CI) by about 16 to $25 \%$ than that for F- IMRT $(p=0)$. VMAT significantly improved CI than I-IMRT ( $\mathrm{p}=0.018$ ) by about $4.5 \%$ for $6 \mathrm{MV}$ and $(\mathrm{p}=0.001)$ for $10 \mathrm{MV}$ by about $6.7 \%$. There were no significant differences between all five plans for homogeneity index (HI).Comparing monitor units (MU) for different I-IMRT plans showed significant increase to MU for $10 \mathrm{MV}$ than that for $6 \mathrm{MV}$ but for VMAT changing energy do not affect MU. MU for $6 \mathrm{MV}$ plans showed an increase in VMAT plans than that for I-IMRT. The MU for I- IMRT and VMAT plans significantly increased compared with that for F-IMRTby about 2.2 to 3.4 times.DVH Comparison between plans shown in figure (2)

Egypt. J. Biophys. Biomed. Eng., Vol. 22,No.1 (2021) 


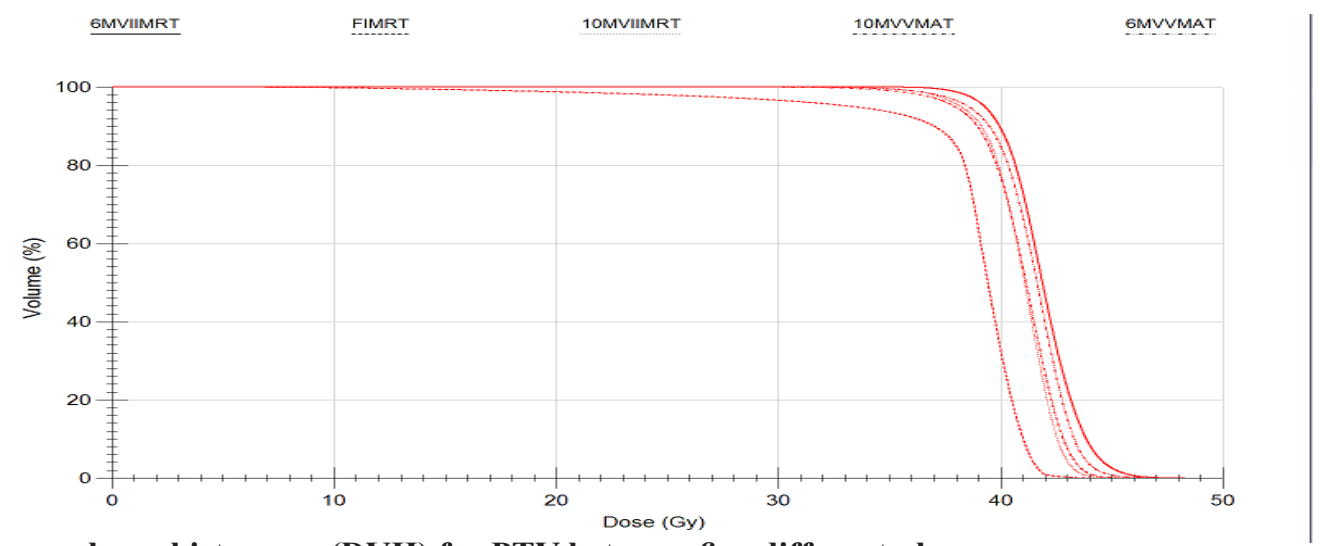

Fig.2.Dose volume histogram (DVH) for PTV between five different plans.

\section{Organs at risk}

Left lung.

The mean value of all left lung parameters (V20 -V10 -V5)Gy are illustrated in Table (3) For high dose level, both I- IMRT and VMAT plans significantly reduced the volume that received less than twenty gray(V20Gy)by about $(31-49 \%)(p=0)$ than F-IMRT. 6MV VMAT significantly reduced V20 Gymore than 6MV IIMRT ( $p=0.001$ ) by about $25 \%$. Varying energies for I- IMRT had a non-significant impact on V20 $(\mathrm{p}=0.593)$ but increased significantly using $10 \mathrm{MV}$ VMAT more than 6MVVMAT $(25 \%)(\mathrm{p}=0.005)$. So inverse planning generally reduced volume receives high dose and using higher energy for VMAT increases it than low energy. For intermediate dose level V 10 Gy: volume received less than ten grays, there was a non-significant difference among I- IMRT and VMAT plans and F- IMRT plan. There was a significant increase (27\%) in V10Gy using $10 \mathrm{MV}$ VMAT over 6MV VMAT $(\mathrm{P}=0.002)$. The same behavior between (6-10 MV) I- IMRT (17\%) but nearly significant $(\mathrm{p}=0.07)$. Theinverse planning does notaffect V10 Gycomparing to Forward planning and using higher energy for inverse planning increase it.For low dose level, 5 Gy volume received less than 5 grays- all plans significantly increased V5 Gyby $(40-80 \%)$ as compared to F- IMRT $(\mathrm{p}=0)$. 6MV VMAT significantly increased volume received 5 Gy than 6MV I- IMRT plan ( $p=0.042)$, the same behavior was also shown for $10 \mathrm{MV}(\mathrm{P}=0)$. $\mathrm{DVH}$ comparison for left lung is shown in figure (3).

\section{Right lung V5Gy}

There was no 5 Gy scattered dose due to the use of F-IMRT planning but using the inverse plan techniques increased this parameter significantly as shown in figure (4). There was a significant increase to V5 Gy for VMAT relative to I-IMRT either with 6 or $10 \mathrm{MV}(\mathrm{p}=0)$ by about three times. The difference between energies was nearly significant for I-IMRT $(\mathrm{p}=0.07)$ and significant for VMAT ( $\mathrm{p}=0$ ) by about 1.7 to 2.2 times using $10 \mathrm{MV}$ more than 6MV. Theinverse planning generally increased scattered low doses to the right lung and using higher energy increases this problem over lower energy. The mean values for the right lung V5Gyare shown in table (4)

\section{The heart}

The mean values of all heart parameters (mean dose - V10 Gy-D5\%) are shown in table (5). There were non-significant differences between the mean values of V10 Gy: volume of heart receiving 10 Gyfor all five plans. The two plans 6 MV I-IMRT $(\mathrm{p}=0.063)$ and VMAT $(\mathrm{P}=0.004)$ showed some significant decreases for the dose received by $5 \%$ of heart volume (D 5\%) compared to F-IMRT plan by about (20-32\%). The difference in D5\% was non-significant for $10 \mathrm{MV}$ (I-IMRT, VMAT) plans compared to F-IMRT. The difference between 6 and $10 \mathrm{MV}$ plans wasnonsignificant either with I- IMRT or VMAT.So, 6 $\mathrm{MV}$ inverse planning reduced dose delivered to $5 \%$ volume of heart but $10 \mathrm{MV}$ inverse planning increased it againby about (7-24\%). The mean heart dose for both I-IMRT and VMAT plans increase the mean heart dose shows a significant increase compared to that of F-IMRT by about $(40 \%)$.There were non-significant differences for mean doses between the four plans 6 and $10 \mathrm{MV}$ I-IMRT and VMAT, either for different techniques or different energies. DVH comparison between plans is shown in figure (5).

\section{Contralateral breast}

The maximum dose for contralateral breast 
TABLE 3.left lung doses comparison between different plans.

\begin{tabular}{cccccc}
\hline & 6MV I-IMRT & 10MV I-IMRT & 6MV VMAT & 10MV VMAT & F -IMRT \\
\hline $\begin{array}{c}\text { Left Lung } \\
\text { (V20 GY) } \%\end{array}$ & $11.45 \pm 0.57$ & $11.93 \pm 0.566$ & $8.49 \pm 0.589$ & $11 \pm 0.64$ & $16.66 \pm 0.75$ \\
Left Lung & $19.53 \pm 0.77$ & $22.79 \pm 1.19$ & $20.58 \pm 1.147$ & $26.34 \pm 1.7$ & $21.9 \pm 0.91$ \\
$\begin{array}{c}\text { (V10 GY })^{\mathrm{a} \%} \\
\text { Left Lung (V5 }\end{array}$ & $39.7 \pm 1.3$ & $42.8 \pm 1.92$ & $44.9 \pm 2.16$ & $50.9 \pm 2.12$ & $28.4 \pm 1.15$ \\
GY) $\%$ & & & & \\
\hline
\end{tabular}
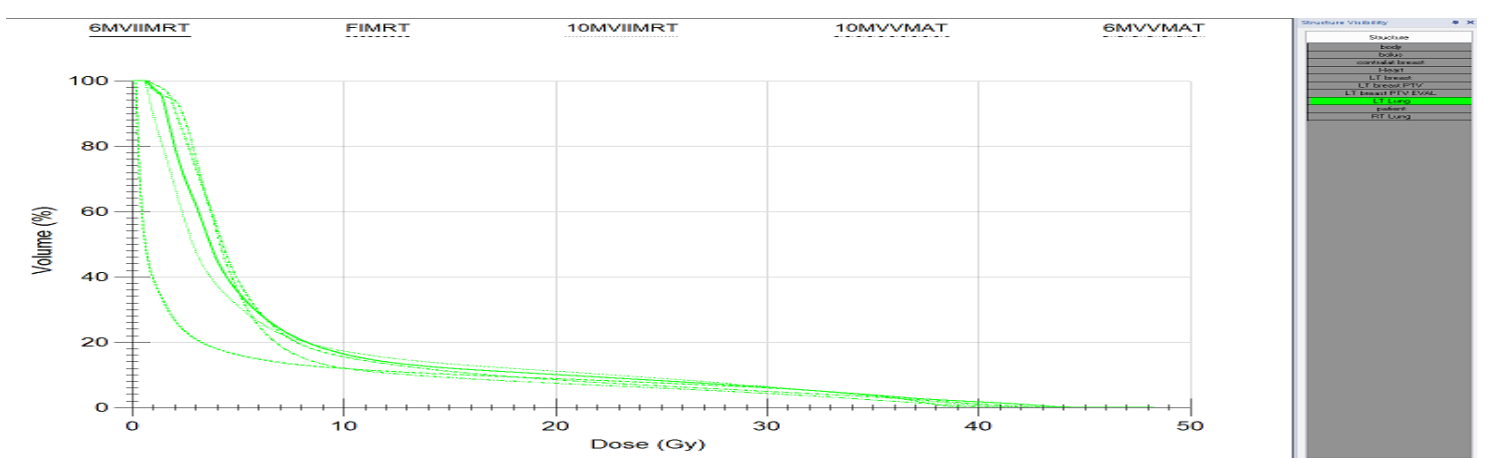

Fig.3.DVD of left lung between five plans

TABLE 4.Mean values for the right lung V5\% (a- percent volume receiving 5 GY)

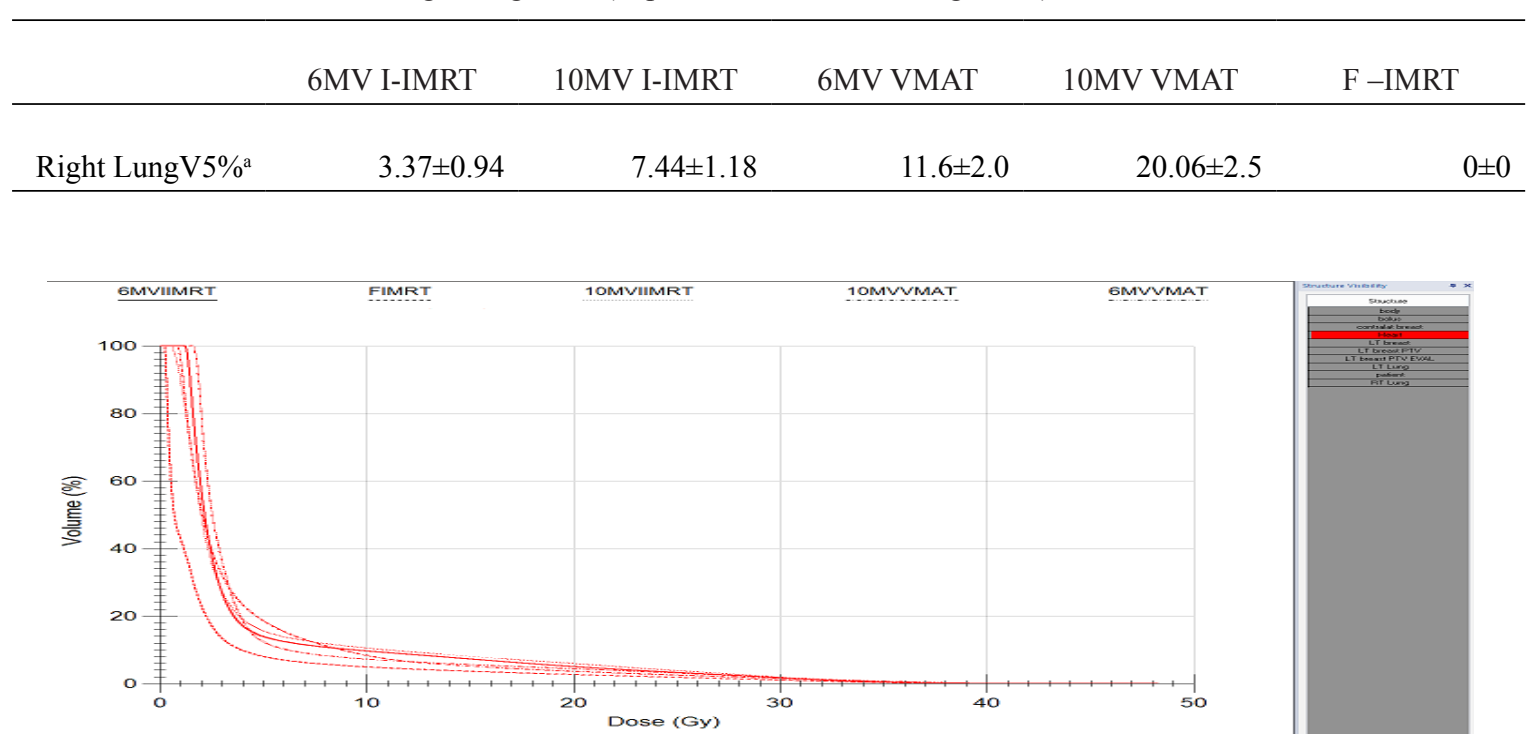

Fig.5. Dose volume histogram (DVH) for heart between five different plans. 


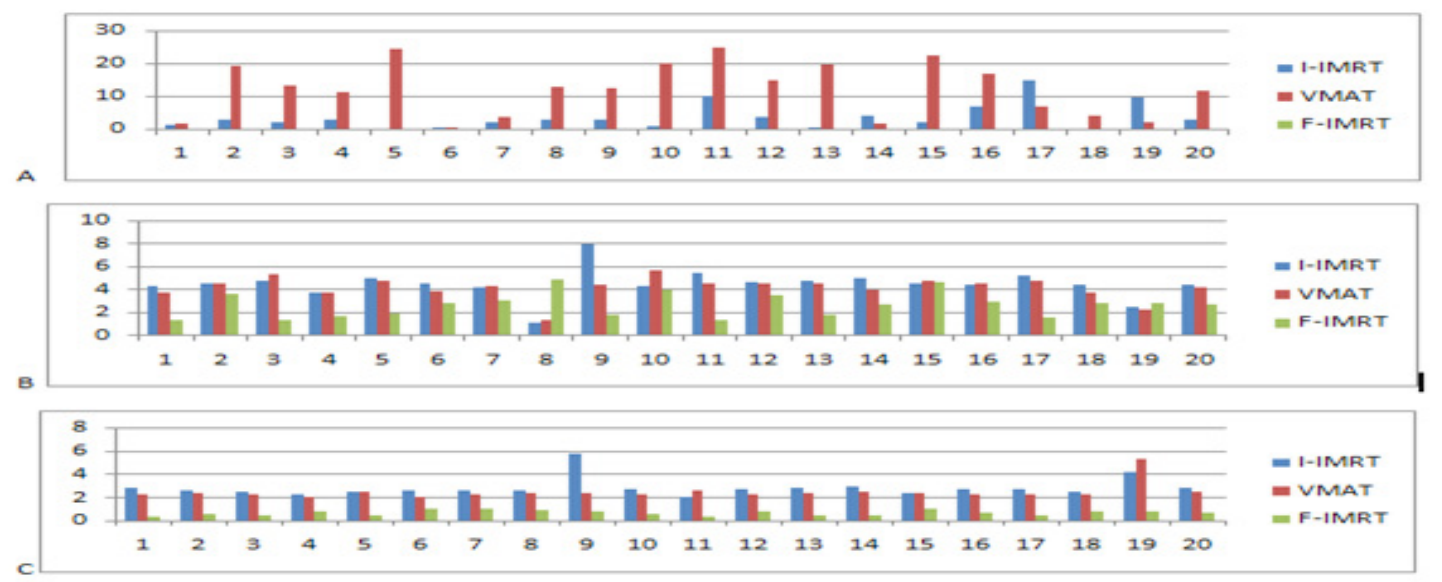

Fig.4. change of A-right lung V5GY x, B-contralateral breast Dmax and C-D5\% for different techniques for randomly selected twenty breast cancer patients. (X-percent volume receiving $\mathbf{x}$ dose, $\mathbf{y}-$ maximum dose, $\mathrm{z}$ - dose delivered to $5 \%$ volume).

TABLE 5. Mean values of all heart parameters (mean dose - V10 GY -D5\%)(a),Percent volume receiving 10 Gy (b), Dose delivered to $\mathrm{x}$ percent volume

\begin{tabular}{cccc}
\hline & 6MV I-IMRT & 10MV I-IMRT & 6MV VMAT \\
\hline Heart V10 GY \%a & $6.05 \pm 0.41$ & $7.4 \pm 0.42$ & $6.08 \pm 0.868$ \\
Heart D5\% Gy & $13.07 \pm 1.005$ & $14.2 \pm 0.957$ & \\
Heart Mean dose GY & $4.18 \pm 0.114$ & $4.23 \pm 0.118$ & $4.1 \pm 0.126$ \\
\hline
\end{tabular}

increased significantly for both I-IMRT and VMAT plans at $6 \mathrm{MV}$ and $10 \mathrm{MV}$ compared to that of F-IMRT plans by about (55-78\%). There were non-significant differences between I-IMRT and VMAT plans with respect to technique or energy. Similar behavior was noticed for contralateral breast (D5\%) by about three times over F-IMRT. Comparison between twenty patients seen in figure (4-b). The mean values of all contralateral breast parameters (maximum dose - D5\%) are shown in table (6) .

\section{Discussion}

From the present study, it is shown that I-IMRT and VMAT improved V95 \% by about $(2-4 \%)$ and using higher energy has no extra benefit either on coverage. Using these advanced techniques increased volume of hot spot and using higher energy do not affect significantly either increase or decrease of D2\% within PTVfigure(6). Generally, inverse planning increases hot spot compared to forward planning but finally homogeneity index (HI) change between different techniques is not obvious. This behavior of HI results from increase of $\mathrm{D} 2 \%$ balanced by

Egypt. J. Biophys. Biomed. Eng., Vol. 22,No.1 (2021) increase of D98\% according to ICRU formula of HI. Many authors showed that inverse planning improved homogeneity usingnumber of fields more than 5(Pasler et al ; 2013, Ayata et al ; 2011, Liu et al ; 2015, Jin et al ; 2013). Conformity index generally improved ininverse planning than forward planning figure (1), this was also shown by( Ayata et al ; 2011 ) and (Supakalin et al ; 2018)at the cost of doubling monitor units that increases scattered low dose to healthy tissues and increases the probability of inducing new cancers (Ayata et al ; 2011 ). For the left lung, inverse planning I-IMRT and VMAT reduced volume receiving high dose than forward F-IMRT as previously demonstrated by (Ayata et al ;2011) and ( Jine $\mathrm{t}$ al ; 2013 ). So inverse planning may result reduction of acute radiation effect. Variation of treatment energy does not affect the volume of left lung receiving high dose. Different techniques give the same range of V10 but using higher energy for inverse planning can increasevolume receiving 10 Gyby about (17-27\%). For low dose region, inverse planning generally increases irradiated volume of low dosesby about (40-80\%) as has been shown in ( Shiau et al;2014 ). This increase may induce lung cancer as a late effect 
TABLE 6. The mean values of all contralateral breast parameters (maximum dose and D5\%). a)Maximum dose, b) Dose delivered to 5 percent volume )

\begin{tabular}{|c|c|c|c|c|c|}
\hline & 6MVI-IMRT & 10MV I-IMRT & 6MV VMAT & 10MV VMAT & F-IMRT \\
\hline Contralateral breast D5\% Gy & $2.83 \pm 0.191$ & $2.41 \pm 0.276$ & $2.46 \pm 0.16$ & $2.14 \pm 0.084$ & $0.657 \pm 0.05$ \\
\hline
\end{tabular}

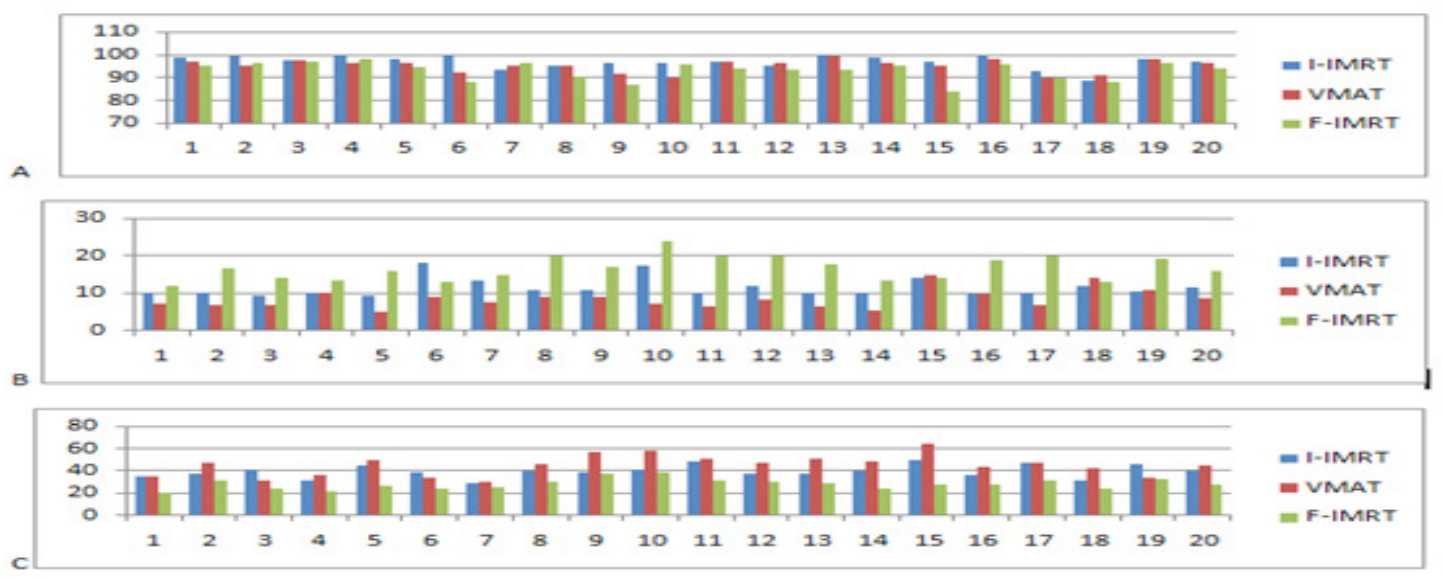

Fig. 6. Change of A-PTV V 95\% $\%^{x}$, B-left lung V20y, C-left lung V5 $5^{y}$ for different techniques for randomly selected twenty breast cancer patients ( $x$-volume of PTV receiving $95 \%$ isodose line, $y$-percent volume receiving $x$ dose).

of radiation. This is a benefit of forward planning over inverse planning in case of other dosimetric parameters are within tolerance. This increase in low doses volumes because inverse planning depends on beam entrance from high number of angles, similarly VMAT increases low doses compared to I-IMRT because all angles in arc used included in plan optimization deliver doses.

For right lung, inverse planning generally results in more irradiated volume at low dose V5 Gy compared to F-IMRT as shown by (Ayataet al ; 2011 ). Inverse planning at $10 \mathrm{MV}$ versus 6 MV doubles scattered low dose either for I-IMRT or VMAT.

For heart doses (V10 Gy) intermediate dose, generally inverse planning shows a non-significant difference compared to F-IMRT as shown in table (6). The only significant improvement is for volume receiving high dose (D5\%)which was reduced using $6 \mathrm{MV}$ I-IMRT and 6MV VMAT over F-IMRT but using 10MV for inverse plans increases D5\% again.Comparing mean heart doses for different techniques show around $40 \%$ increase for I-IMRT and VMAT compared to F-IMRT( Ercan et al ;2010, Karpf et al ; 2019 ) .
For contralateral breast Dmax is doubled for I-IMRT and VMAT relative to F-IMRT as shown in table (7). Contralateral breast D5\% increased around 3 times using inverse planning more than forward planning as shown in figure (4).

\section{Conclusion}

I-IMRT and VMAT planning improve PTV coverage, conformity index and maintain acceptable level of homogeneity. This works well for reducing high dose levels for organs at risk at the cost of increasing scattered low doses to lung, heart, and contralateral breast. Using high energy for inverse planning may results in increasing the volume of scattered low and intermediate dose levels, especially with VMAT, because VMAT increases the number of monitor units up to three times in addition to the global irradiated volume. According to the results, the trade-off between techniques depends on case anatomy. Inverse planning works well with complex shapes to reduce acute radiation side effect and maintain high level of tumor control (Henry et al ; 2018 , Lauche et al ; 2016 ), but this increases the risk of late radiation effect ( Morgan et al ; 2012).From results F-IMRT planning may be the first choice in

Egypt. J. Biophys. Biomed. Eng., Vol. 22,No.1 (2021) 
case of high dose tolerances have been achieved. The choice of F-IMRT to avoid scattering of low doses. In case there are high dose tolerances not achieved by F-IMRT specially with complex breast shape so inverse planning can be used with caution.However, the relation between technique and PTV shape needs further investigation for larger number of patients' sample to study the relation between breast concavity angle, depth of heart, and the volume lung inside breast concavity and scattered low doses to OARs(Shiau et al ; 2014).

\section{References}

1. Arbyn, M., Weiderpass, E., Bruni, L., de Sanjosé, S., Saraiya, M., Ferlay, J., \& Bray, F. (2020). Estimates of incidence and mortality of cervical cancer in 2018: a worldwide analysis. The Lancet Global Health, 8(2), e191-e203.

2. Arsene-Henry, A., Foy, J. P., Robilliard, M., Xu, H. P., Bazire, L., Peurien, D., ... \& Kirova, Y. M. (2018). The use of helical tomotherapy in the treatment of early stage breast cancer: indications, tolerance, efficacy - a single center experience. Oncotarget, 9(34), 23608.

3. Avigo, C; Mignogna, M., \&Linslata, S. (2017). EP-1759: MLC positioning study based on EPID images analyzed with the Dosimetry Check software. Radiotherapy and Oncology, 123, S968-S969.

4. Ayata, H. B; Güden, M; Ceylan, C; Kücük, $\mathrm{N}$;\&Engin, K. (2011). Comparison of dose distributions and organs at risk (OAR) doses in conventional tangential technique (CTT) and IMRT plans with different numbers of beam in left-sided breast cancer. Reports of Practical Oncology \& Radiotherapy, 16(3), 95-102.

5. Chen, G. P., Liu, F., White, J., Vicini, F. A., Freedman, G. M., Arthur, D. W., \& Li, X. A. (2015). A planning comparison of 7 irradiation options allowed in RTOG 1005 for early-stage breast cancer. Medical Dosimetry, 40(1), 21-25.

6. Ercan, T; İğdem, Ş ;Alço, G., Zengin, F ; Atilla, S ; Dinçer, M ;\&Okkan, S. (2010). Dosimetric comparison of field in field intensity-modulated radiotherapy technique with conformal radiotherapy techniques in breast cancer. Japanese journal of radiology, 28(4), 283-289.

7. Dibden,A.,Offman,J,S.w.,\&Gabe,R.(2020) .Worldwide review and meta-analysis of cohort studies measuring the effect of mammography screening programmes on incidence -based breast cancer mortality .Cancers ,12(4),976.ISO690

8. Henson, K. E ;McGale, P ; Taylor, C; \& Darby, S. C. (2013). Radiation-related mortality from heart disease and lung cancer more than 20 years after radiotherapy for breast cancer. British journal of cancer, 108(1), 179.

9. Hooning, M. J ;Botma, A ; Aleman, B. M ; Baaijens, M. H ; Bartelink, H ; Klijn, J. G ; ... \& Van Leeuwen, F. E. (2007). Long-term risk of cardiovascular disease in 10-year survivors of breast cancer. Journal of the National Cancer Institute, 99(5), 365-375.

10. Jabbari K (2011). Review of fast monte carlo codes for dose calculation in radiation therapy treatment planning. J Med Signals Sens, 1, 73-86.

11. Jin, G. H ; Chen, L. X ; Deng, X. W ; Liu, X. W ; Huang, Y ; \& Huang, X. B. (2013). A comparative dosimetric study for treating left-sided breast cancer for small breast size using five different radiotherapy techniques: conventional tangential field, filed-in-filed, tangential-IMRT, multi-beam IMRT and VMAT. Radiation oncology, 8(1), 89.

12. Karpf, D; Sakka, M ; Metzger ; \&Grabenbaur,G,G (2019) )left breast irradiation with tangentional intensity modulated radiotherapy ( $\mathrm{t}$ - IMRT ) versus tangentional volumetric modulated arc therapy ( $t$ - VMAT ) : trade - offs between secondary cancer risk induction risk and optimal target coverage . Radiation oncology (2019) 14: 156.

13. Lauche, O., Kirova, Y. M., Fenoglietto, P., Costa, E., Lemanski, C., Bourgier, C., ... \&Azria, D. (2016). Helical tomotherapy and volumetric modulated arc therapy: new therapeutic arms in the breast cancer radiotherapy. World Journal of Radiology, 8(8), 735.

14. Liu, Z. M ; Ge, X. L ; Chen, J. Y ; Wang, P. P ; Zhang, C ; Yang, X ; ... \& Lu, J. (2015). Adjuvant radiotherapy after breast conserving treatment for breast cancer: A dosimetric comparison between volumetric modulated arc therapy and intensity modulated radiotherapy. Asian Pac $J$ Cancer Prev, 16, 3257-65.

15. McLaughlin, S. A ; Ochoa-Frongia, L. M; Patil, S. M ; Cody III, H. S ; \& Sclafani, L. M. (2008). Influence of frozen-section analysis of sentinel lymph node and lumpectomy margin status on reoperation rates in patients undergoing breastconservation therapy. Journal of the American College of Surgeons, 206(1), 76-82. 
16. Morgan, E. A ;Kozono, D. E ; Wang, Q., Mery, C. M ; Butrynski, J. E ; Baldini, E. H; ... \& Raut, C. P. (2012). Cutaneous radiation-associated angiosarcoma of the breast: poor prognosis in a rare secondary malignancy. Annals of surgical oncology, 19(12), 3801-3808.

17. Narayanasamy, G; Granatowicz, D; Baacke, D., Li, Y; Gutierrez, A; Papanikolaou, N; \& Stathakis, S. (2015). AComparison between Three-Dimensional Conformal Radiotherapy, Intensity-Modulated Radiotherapy, and Volumetric-Modulated Arc Therapy Techniques for Stereotactic Body Radiotherapy of Lung Tumors. International Journal of Medical Physics, Clinical Engineering and Radiation Oncology, 4(02), 104.

18. Pasler, M; Georg, D; Bartelt, S; \&Lutterbach, J. (2013). Node-positive left-sided breast cancer: does VMAT improve treatment plan quality with respect to IMRT?. Strahlentherapie und Onkologie, 189(5), 380-386.

19. Rangaraj, D; Oddiraju, S ; Sun, B; Santanam, L; Yang, D; Goddu,\&Papiez, L. (2010). Fundamental properties of the delivery of volumetric modulated arc therapy (VMAT) to static patient anatomy. Medical physics, 37(8), 4056-4067.

20. Sardaro, A; Petruzzelli, M. F; D’Errico, M. P; Grimaldi, L; Pili, G; \&Portaluri, M. (2012). Radiation-induced cardiac damage in early left breast cancer patients: risk factors, biological mechanisms, radiobiology, and dosimetric constraints. Radiotherapy and Oncology, 103(2), 133-142.

21. Sung, H., Ferlay, J., Siegel, R. L., Laversanne, M., Soerjomataram, I., Jemal, A., \& Bray, F. (2021). Global cancer statistics 2020: GLOBOCAN estimates of incidence and mortality worldwide for 36 cancers in 185 countries. $C A$ : a cancer journal for clinicians.

22. Shiau, A. C; Hsieh, C. H; Tien, H. J; Yeh, H. P; Lin, C. T; Shueng, P. W; \& Wu, L. J. (2014). Leftsided whole breast irradiation with hybrid-IMRT and helical tomotherapydosimetriccomparison .Hindawi publishing corporation Bio Med research international, volume 2014.

23. Simões, R; Rozendaal, R; Trinks, J; Kalisvaart, R; Van der Heide, U; \&Remeijer, P. (2018). EP2123: Clinical evaluation of an auto-segmentation toolbox for breast CTV. Radiotherapy and Oncology, 127, S1168-S1169.
25. Supakalin, N; Pesee, M; Thamronganantasakul, K; Promsensa, K; Supaadirek, C; \&Krusun, S. (2018). Comparision of Different Radiotherapy Planning Techniques for Breast Cancer after Breast Conserving Surgery. Asian Pacific journal of cancer prevention: APJCP, 19(10), 2929.

26. Woon, Y. L; Heng, S. P; Wong, J. H. D; \& Ung, N. M. (2016, March). Comparison of selected dose calculation algorithms in radiotherapy treatment planning for tissues with in homogeneities. In Journal of Physics: Conference Series(Vol. 694, No. 1, p. 012024). IOP Publishing.

27. https://www.icru.org/home/reports/prescribingrecording-and-reporting-photon-beam-therapyreport-50

24. 


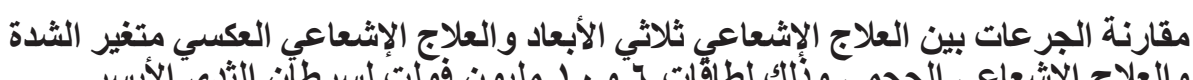

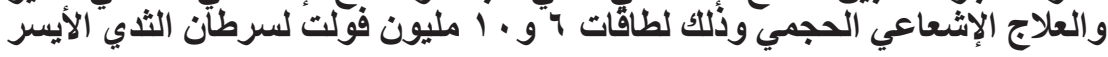

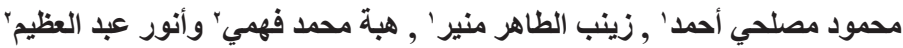

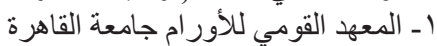

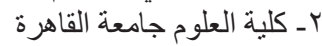

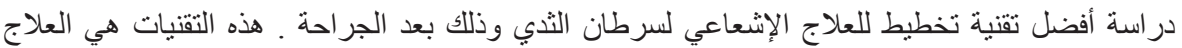

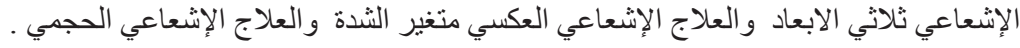

في هذه الدر اسة يتم مقارنة الجر عات بين اربع خطط علاجية متغيرة الثدة بشكل عكسي وخطة تقليدية ثلاثية

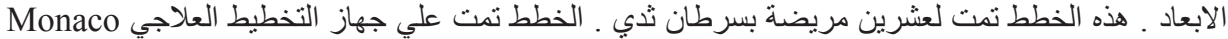

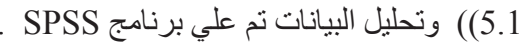

حقق التخطيط العكسي تغطية أفضل من العلاج الإشعاعي التقليدي (p=0.001, 00.7) و وتحسن في تشكيل

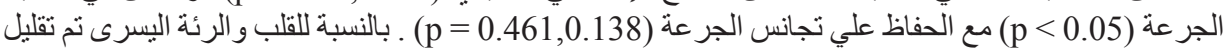

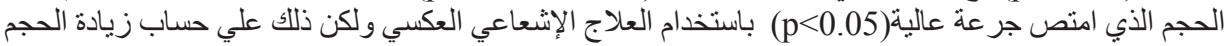

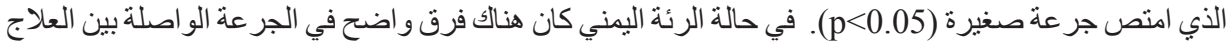

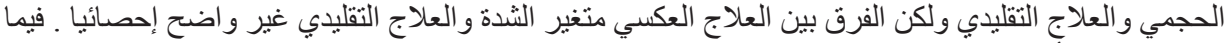

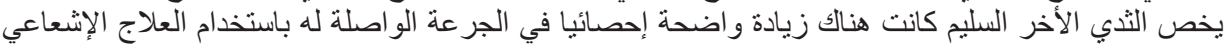
العكسي عن العلاج ثلاثي الابعاد الانيم

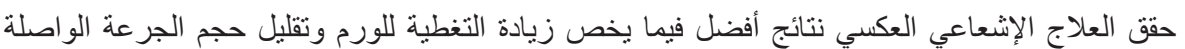

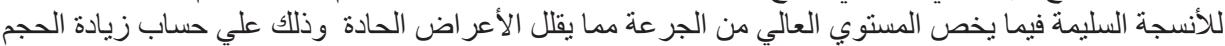

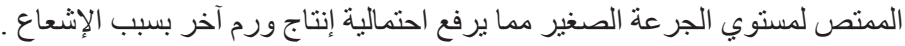

